\title{
Cardiac Remodeling and MMPs on the Model of Chronic Daunorubicin-Induced Cardiomyopathy in Rabbits
}

\author{
M. ADAMCOVÁ ${ }^{1}$, A. POTÁČOVÁ ${ }^{1}$, O. POPELOVÁ ${ }^{2}$, M. ŠTĚRBA ${ }^{2}$, Y. MAZUROVÁ ${ }^{3}$, \\ H. AUPPERLE ${ }^{4}$, V. GERŠL ${ }^{2}$ \\ ${ }^{1}$ Department of Physiology, ${ }^{2}$ Department of Pharmacology, ${ }^{3}$ Department of Histology and \\ Embryology, Charles University in Prague, Faculty of Medicine in Hradec Králové, Hradec \\ Králové, Czech Republic, ${ }^{4}$ Institut für Veterinär-Pathologie, Universität Leipzig, Germany
}

Received March 30, 2009

Accepted December 18, 2009

On-line April 20, 2010

\section{Summary}

The matrix metalloproteinases (MMPs) play a key role during cardiac remodeling. The aim of the study was to investigate the changes in collagenous proteins and MMPs in the model of non-ischemic, anthracycline-induced chronic cardiomyopathy in rabbits using both biochemical and histological approaches. The study was carried out in three groups of Chinchilla male rabbits: 1) daunorubicin (3 mg/kg, once weekly for 10 weeks), 2) control (saline in the same schedule), 3) daunorubicin with the cardioprotectant dexrazoxane $(60 \mathrm{mg} / \mathrm{kg}$, before each daunorubicin). Morphological changes in the myocardium of daunorubicin-treated animals were characterized by focal myocardial interstitial fibrosis of different intensity. The subsequent proliferation of the fibrotic tissue was marked by an increased content of both collagen types I and III, which resulted in their typical coexpression in the majority of bundles of fibers forming either smaller or larger scars. Biochemical analysis showed a significantly increased concentration of hydroxyproline, mainly in the pepsin-insoluble fraction of collagenous proteins, in the daunorubicin-treated group $(1.42 \pm 0.12 \mathrm{mg} / \mathrm{g})$ as compared with the control $(1.03 \pm 0.04 \mathrm{mg} / \mathrm{g})$ and dexrazoxane $(1.07 \pm 0.07$ $\mathrm{mg} / \mathrm{g}$ ) groups. Dexrazoxane co-administration remarkably reduced the cardiotoxic effects of daunorubicin to the extent comparable with the controls in all evaluated parameters. Using zymography, it was possible to detect only a gelatinolytic band corresponding to MMP-2 (MMP-9 activity was not detectable). However, no significant changes in MMP-2 activity were determined between individual groups. Immunohistochemical analysis revealed increased MMP-2 expression in both cardiomyocytes and fibroblasts. Thus, this study has revealed specific alterations in the collagen network in chronic anthracycline cardiotoxicity in relationship to the expression and activity of major MMPs.

\section{Key words}

Anthracyclines - Cardiomyopathy - Remodeling • Collagen • Matrix metalloproteinases

\section{Corresponding author}

Michaela Adamcová, Department of Physiology, Charles University in Prague, Faculty of Medicine in Hradec Králové, Šimkova 870, 50038 Hradec Králové, Czech Republic. E-mail: adamcova@lfhk.cuni.cz

Cardiac toxicity associated with repeated administration of anthracyclines represents a serious complication of their use in anticancer chemotherapy. So far, dexrazoxane is the only drug which has been clearly demonstrated to protect the myocardium from anthracycline-induced toxicity both in experimental and clinical settings (Herman et al. 1994, Marty 2006). Besides apparent clinical implications, anthracyclineinduced cardiotoxicity is often used as a very useful experimental model of non-ischemic cardiomyopathy and congestive heart failure.

The development of heart failure is a chronic process associated with myocardial remodeling which 
involves necrosis and/or apoptosis of cardiomyocytes, proliferation of fibroblasts and alteration of both intracellular components of the myocardium, as well as of major structural proteins of the extracellular matrix (ECM) (Graham et al. 2008). Current understanding to the ECM remodeling attributes a pivotal role to the family of matrix metalloproteinases (MMP) (Spinale 2007). However, recent studies have shown that MMPs cleave not only the key protein components of the myocardial extracellular compartment but also numerous sarcomeric and cytoskeletal proteins within the cardiac myocytes (e.g., troponins, MLC-1 or desmin) (Schulz 2007). The structural damage of cardiomyocytes can be estimated by the release of cardiac biomarkers into the plasma. It has been previously shown that cardiac troponins may serve as useful biomarkers of anthracycline-induced cardiac damage and they therefore become gradually utilized in clinical practice (Adamcová et al. 2005, Adamcová et al. 2007). However, a little attention has been paid to the alterations of the extracellular matrix and the involvement of MMPs in anthracycline cardiotoxicity yet.

Hence, this study aimed to assess the changes in MMPs along with the determination of quantitative and qualitative alterations in the expression of collagenous proteins during cardiac remodeling induced by repeated administration of daunorubicin in rabbits.

Chronic anthracycline cardiomyopathy was induced in Chinchilla male rabbits using a well described model (Geršl and Hrdina 1994, Šimůnek et al. 2004) daunorubicin (DAU, $3 \mathrm{mg} / \mathrm{kg}$, i.v., Daunoblastina, Pharmacia Italia, Italy, $\mathrm{n}=11$ ). Control animals received saline $(1 \mathrm{ml} / \mathrm{kg}$, i.v., $\mathrm{n}=8)$ in the same schedule. The third group of animals received a model cardioprotectant dexrazoxane (60 mg/kg, i.p., Cardioxane, Novartis, Switzerland) $30 \mathrm{~min}$ before each DAU administration. At the end of the experiment, invasive hemodynamic measurement $\left(\mathrm{dP} / \mathrm{dt}_{\max }\right)$ was performed using a Micro-Tip pressure catheter (2.3F, Millar Instruments, U.S.A.) connected to a data acquisition system Powerlab and a Chart 5.4.2 software (ADInstruments Pty., Australia). The concentrations of cardiac troponin $\mathrm{T}(\mathrm{cTnT})$ were measured using an Elecsys Troponin $\mathrm{T}$ STAT Immunoassay (Roche Diagnostics, Switzerland). Cardiac troponin concentrations over the whole experimental period were expressed as medians of the area under the curve (AUC). For drug administration, blood sampling and other mini-invasive/non-invasive operations, a combination of short-acting anesthetic agents was used (midazolam $1.25 \mathrm{mg} / \mathrm{kg}+$ ketamine $50 \mathrm{mg} / \mathrm{kg}$, i.m.). Surgical anesthesia for the preparations and invasive hemodynamic measurements performed at the end of the experiment was induced with pentobarbital $(30 \mathrm{mg} / \mathrm{kg}$, i.v.). All the experiments were performed in accordance with the Guide for the Care and Use of Laboratory Animals published by the US National Institutes of Health (NIH Publication, 1996).

Biochemical analysis of collagenous proteins was performed as described previously (Pelouch et al. 1995). Hydroxyproline concentrations were measured spectrophotometrically at $550 \mathrm{~nm}$ in both pepsin-soluble and pepsin-insoluble fractions. In addition, proteins were separated by $7.5 \%$ SDS-PAGE and detected using silver staining. MMP enzyme activities were assayed by zymography using either gelatine (Type A, SigmaAldrich) or casein ( $\beta$-casein, Sigma-Aldrich) or collagen (Collagen Type I, Sigma-Aldrich) as MMPs substrates. The left ventricular samples were homogenized in the lysis buffer containing cacodylic acid, $\mathrm{NaCl}, \mathrm{CaCl}_{2}$, $\mathrm{NaN}_{3}$, Triton X-100, and $\mathrm{ZnCl}_{2}$.

Histological examination was made on paraffin transversal sections of the heart ventricles stained with hematoxylin-eosin for basic evaluation, while Picro-sirius Red method was employed for collagen examination, particularly in polarized light. Immunohistochemical detection of MMP-2 was performed by standard peroxidase anti-peroxidase (PAP) technique using the primary rabbit anti-MMP-2 (Neomarkers, CA, USA; dilution 1:400) antibody and the swine anti-rabbit $\mathrm{IgG}$ (DAKO, Denmark; dilution 1:100) secondary antibody.

Statistical evaluation was performed with statistical softwares SigmaStat 3.5 (SPSS, U.S.A.) and NCSS 2004 (NCSS, U.S.A.). Significances were assessed with one-way ANOVA or Kruskal-Wallis ANOVA on ranks (with Bonferroni post-hoc analysis).

Invasive examination of the left ventricular systolic function $\left(\mathrm{dP} / \mathrm{dt}_{\max }\right)$ revealed progressive and significant impairment in the daunorubicin group in comparison with both control and dextrazoxane co-treated animals (control $1158.3 \pm 76.0 \quad \mathrm{kPa} / \mathrm{s}$, $\mathrm{DEX}+\mathrm{DAU} 1174.7 \pm 100.3 \mathrm{kPa} / \mathrm{s}$ vs. DAU $773.6 \pm 46.4$ $\mathrm{kPa} / \mathrm{s}$, one-way ANOVA, $\mathrm{p}<0.05$ ). Concomitantly, plasma concentrations of troponin $\mathrm{T}$ (a biomarker of cardiomyocyte injury) were found to be significantly increased in the DAU-treated animals in comparison with the control and dexrazoxane groups (control $0.003 \mathrm{ng} / \mathrm{ml}$; DEX+DAU 0.000 ng/ml vs. DAU 0.315 ng/ml, KruskalWallis ANOVA, $\mathrm{p}<0.01)$. 

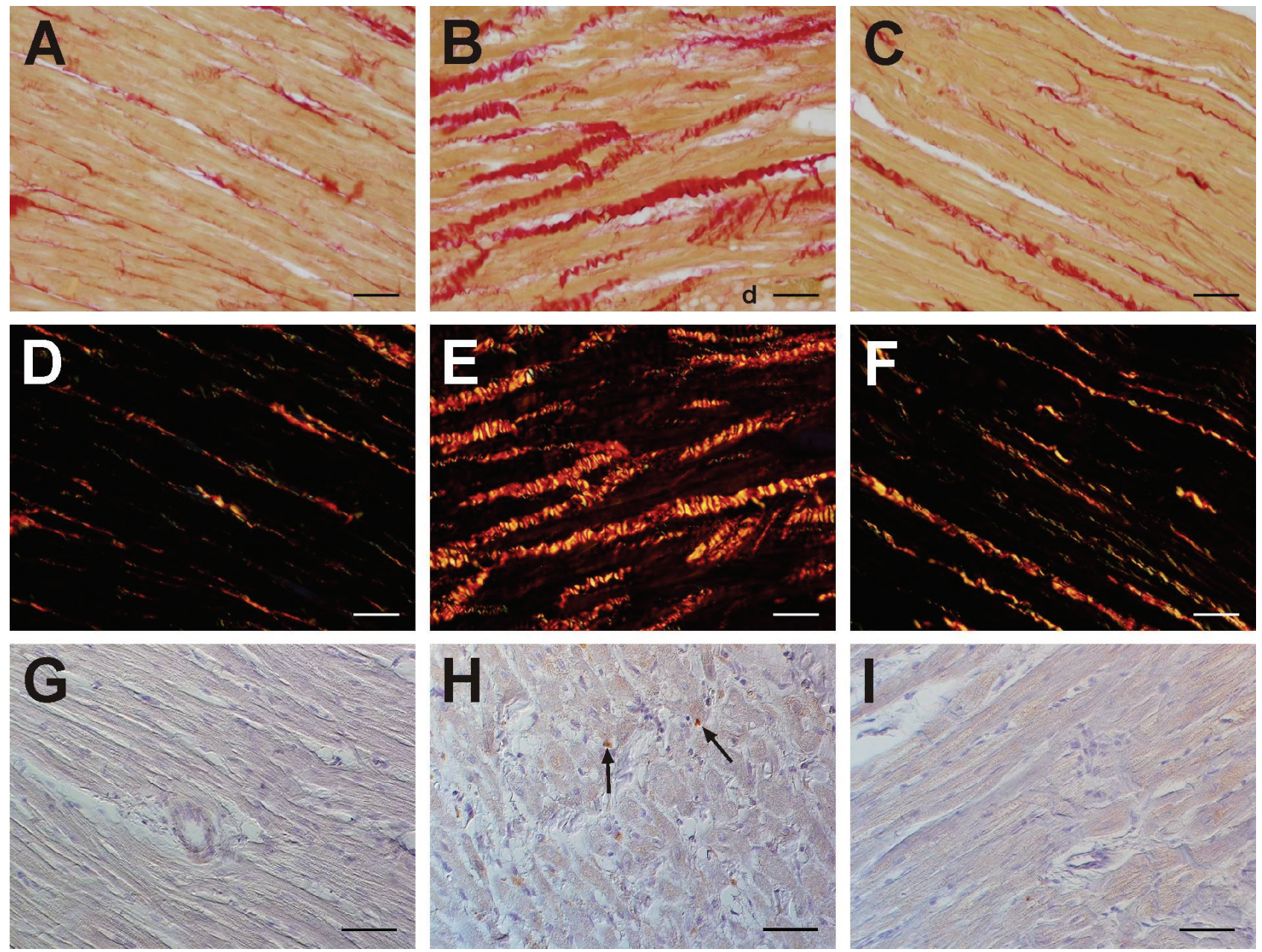

Fig. 1. Myocardium of the left ventricle wall. A,D,G. Control group - only fine loose connective tissue (A - red) with vessels, composed of fibres formed of both collagen type I (D - yellow/red) and III (D - green), surrounds the columns of cardiomyocytes (A - pale yellow); MMP-2 expression is not detectable (G). B,E,H. DAU group - intensive proliferation of interstitial and above all of perivascular fibrotic tissue (B - red) at first represented mainly by reticular fibres (collagen III), in later stages by collagen fibres (collagen type I E: yellow/red), replaces destroyed cardiomyocytes and results in focal formation of smaller or larger fibrotic scars; (E) "d" degenerated myocyte with markedly vacuolized cytoplasm; $(\mathbf{H})$ the mild expression of MMP-2 is in both myofibrils (cardiomyocytes) and single fibroblasts (arrows). C,F,I. DEX+DAU group - No conspicuous differences are found in comparison with the control group (C,F); the only difference is the mild focal expression of MMP-2 bound to the myofibrils but, similarly to the controls, the fibroblasts are not labeled (I). A-C: Picro-sirius Red staining - bright-field microscopy; D-F: Picro-sirius Red staining - polarization microscopy; G-I: antiMAP-2 peroxidase immunohistochemistry. Bar $-50 \mu \mathrm{m}$

The histological examination of the myocardium of controls revealed a normal morphology. Cardiomyocytes were arranged in typical columns surrounded by the loose interstitial connective tissue with capillaries in which fine reticular fibers, formed by collagen type III, prevailed. The dense connective tissue, composed mainly of thicker wavy bundles of collagen fibers (collagen type I), was arranged around larger vessels (Figs 1A,D). In the DAU group, interstitial myofibrosis developed in places, where focal degeneration (death) of the groups of cardiomyocytes occurred. The reparative process was characterized by the production of both types of collagen, but type III was produced predominately at the beginning of healing, whereas type I collagen was typical of the advanced stages of the process (Figs 1B,E and 2B). Dexrazoxane co-administration apparently prevented the abovedescribed DAU-induced changes (Figs 1C,F), therefore the structure of the myocardium was fully comparable with intact controls (Figs 1A,D).

Biochemical analysis of the myocardium from the DAU group showed a significantly $(p<0.01)$ increased concentration of hydroxyproline mainly in the pepsininsoluble fraction of collagenous proteins $(1.42 \pm 0.12$ $\mathrm{mg} / \mathrm{g}$ of wet tissue) as compared with the control $(1.03 \pm 0.04 \mathrm{mg} / \mathrm{g}$ of wet tissue) and dexrazoxane 

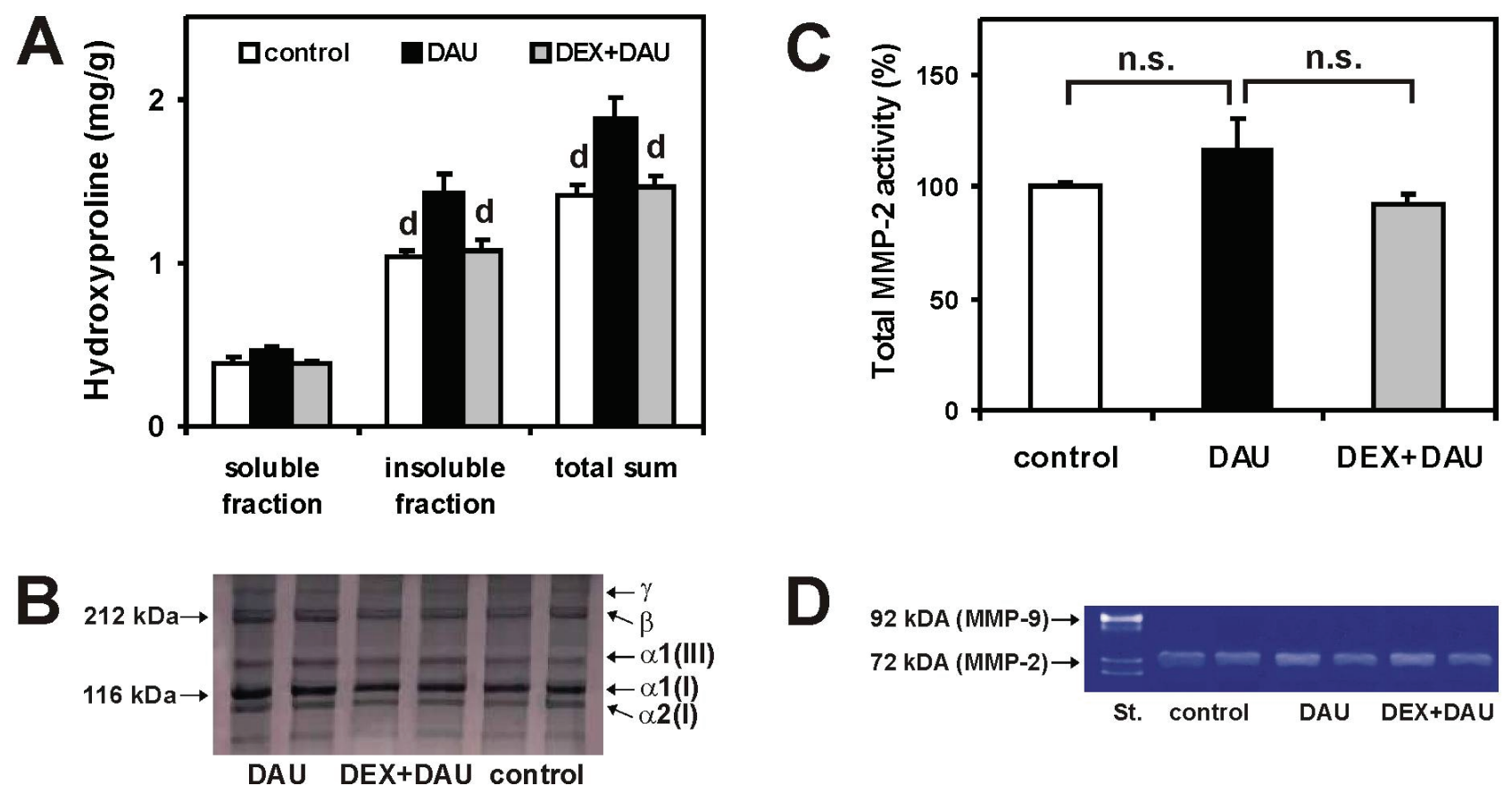

Fig. 2. Biochemical finding. A - Concentration of hydroxyproline in the fraction of pepsin-soluble and insoluble collagenous proteins in $\mathrm{mg} / \mathrm{g}$ of wet tissue. Statistical significances (one-way ANOVA, $\mathrm{P}<0.01$ ) in comparison with "d" daunorubicin group. B - Typical gel electrophoresis profile of collagenous proteins. Typical gel electrophoresis profile $\gamma$ fraction (chain polymers, collagen type I + III), $\beta$ fraction (chain dimmers, collagen type I + III), $\alpha 1=$ mixture of the individual $\alpha 1$ chain (collagens type I + III), $\alpha 2=\alpha 2$ chains (collagen type I). C - Total activity of MMP-2 assessed by zymography. D - Representative gelatinase zymography showing the MMPactivity at the end of the experiment in the comparison with the migration of standards MMP-2/MMP-9 (Chemicon, USA).

$(1.07 \pm 0.07 \mathrm{mg} / \mathrm{g}$ of wet tissue) groups (Fig. 2A). This points on the increased content of predominantly highly cross-linked collagenous aggregates in the DAU group. Using zymography, a predominant band of MMP-2 (72 kDa) was detected in all groups (Fig. 2D), whereas no activity of other MMPs was identified either on the casein or collagen containing zymograms. The average MMP-2 activity at the end of the experiment appeared to be somewhat higher in the DAU group as compared to both control and dexrazoxane groups. However, significant difference was not reached with respect to large interindividual variability of this parameter in the DAU group (Fig. 2C).

In controls, MMP-2 expression was not detectable immunohistochemically (Fig. 1G). However, the DAU treatment resulted in a mild expression of MMP-2 in most of the animals in isolated (sporadic) cardiomyocytes and fibroblasts within the foci with significant toxic damage (Fig. 1H). The expression tended to be more prominent in the animals with most pronounced functional and histopathological changes. MMP-2 expression was detected only in a half of the animals in the dexrazoxane group and the expression was generally mild and restricted to cardiomyocytes (Fig. 1I).

It has been recently revealed that the rabbit is a very suitable model for the study of human heart failure because the biophysical and biochemical characteristics of rabbit myofibres as well as the calcium handling closely resemble the properties of the human cardiomyocytes (Marian 2005). In our study, the histological examination of the left ventricular myocardium showed different stages of focal degeneration of cardiomyocytes in the DAU group. The cytoplasmic vacuolization and loss of myofibrils finally resulted in a total disintegration of the myocytes followed by the development of focal interstitial fibrosis. The newly synthesized collagen contained a greater proportion of the more compliant type III collagen, which is known to form less extensive cross-linking making the replacement collagen functionally inadequate (Graham et al. 2008). However, the collagen type I already prevailed in our samples because of maturation of connective tissue into fibrotic scar during the chronic experiment. The above-described alterations in the collagen network create the basis for the development of typical fibrosis accompanying dilated cardiomyopathy.

MMPs are generally considered to be among the key regulators of extracellular matrix remodeling under pathological conditions (Spinale 2007). It has been shown that MMP-2 and MMP-9 are significantly involved in 
ischemia/reperfusion injury and subsequent myocardial remodeling (Lalu et al. 2005). These MMPs are known to degrade gelatine, collagen type I and III which are the main components of the myocardial extracellular matrix. Similarly, an increased activity of these MMPs has been shown in acute anthracycline toxicity induced by a single supratherapeutical dose (Pacher et al. 2002, Bai et al. 2004). However, involvement of MMPs in the myocardial remodeling associated with a clinicallyrelevant chronic anthracycline cardiomyopathy development remains uncertain. In the present study, with the use of zymography, we were able to detect only one gelatinolytic band which corresponded to MMP-2. However, the quantification of MMP-2 activity did not show any significant changes between the groups in the study. No MMP-9 signal was detected, which may be due to the absence of a marked invasion of neutrophils (Schulz 2007). This finding is consistent with the recent data (Truter et al. 2009) on the extracellular matrix remodeling in the chronic aortic regurgitation model. In this study, fibrosis was also not accompanied by a detectable quantity of MMP-9 at both protein and mRNA levels. The results of our study indicated that both main myocardial cell types (cardiomyocytes and fibroblasts) possess the capacity to express MMP-2 in rabbits. Although non-cardiomyocyte cell types have been particularly implicated as the sites for the synthesis of MMPs within the ECM, it should be noted that $90 \%$ of the myocardial mass is composed of cardiomyocytes (Spinale 2007). Interestingly, no expression of gelatinolytic MMPs was detected in the extracellular matrix, although gelatinases are known to be secreted by cardiac cells into the interstitium. These immunohistochemical findings were similar to those reported by Aupperle et al. (2007) in doxorubicin-treated rabbits, even though the myocardial fibrosis and the degenerative changes in cardiomyocytes were less pronounced.
In the present study, the authors have shown that chronic anthracycline cardiotoxicity was associated with profound changes in the left ventricular morphology and function. These changes were accompanied by evident alterations in the collagen network which corresponds to the typical successive formation of the fibrotic scar tissue. Surprisingly, no significant changes in MMPs activity were found in the myocardium of rabbits with fully developed cardiomyopathy. In this way, our present observations differ from previous reports on anthracycline cardiotoxicity (Pacher et al. 2002, Bai et al. 2004). However, it should be noted that these reports dealt with the acute anthracycline toxicity induced by a single high dose and therefore their translatability to clinically relevant conditions remains uncertain. Although MMP-9 activity is involved in many other cardiac pathologies, our data strongly suggest that MMP9 is not involved in chronic anthracycline cardiotoxicity which might be associated with the absence of typical inflammatory infiltration. However, the investigation of the role of MMPs in the earlier phases of the development of chronic anthracycline cardiomyopathy merits a further study as the expression and activity of MMPs might undergo more dynamic changes in the course of cardiotoxicity development. Importantly, this study shows that the signs of DAU-induced myocardial remodeling could be efficiently prevented by dexrazoxane co-treatment.

\section{Conflict of Interest}

There is no conflict of interest.

\section{Acknowledgements}

This study was supported by the Charles University Grant Agency No. 53107/2007/C and Research Project MSM0021620820.

\section{References}

ADAMCOVÁ M, ŠTĚRBA M, ŠIMU゚NEK T, POTÁČOVÁ A, POPELOVÁ O, MAZUROVÁ Y, GERŠL V: Troponin as a marker of myocardiac damage in drug-induced cardiotoxicity. Expert Opin Drug Saf 4: 457-472, 2005.

ADAMCOVÁ M, ŠIMƯNEK T, KAISEROVÁ H, POPELOVÁ O, ŠTĚRBA M, POTÁČOVÁ A, VÁVROVÁ J, KVASNIČKOVÁ E, GERŠL V: In vitro and in vivo examination of cardiac troponins as biochemical markers of drug-induced cardiotoxicity. Toxicology 237: 218-228, 2007.

AUPPERLE H, GARBADE J, SCHUBERT A, BARTEN M, THEIN S, SHOON HA, MOHR FW: Effect of autologous stem cells on immunohistochemical patterns and gene expression of metalloproteinases and their tissue inhibitors in doxorubicin cardiomyopathy in a rabbit model. Vet Pathol 44: 494-503, 2007. 
BAI P, MABLEY JG, LIAUDET L, VIRÁG L, SZABÓ C, PACHER P: Matrix metalloproteinase activation is an early event in doxorubicin-induced cardiotoxicity. Oncol Rep 11: 505-508, 2004.

GERŠL V, HRDINA R: Noninvasive polygraphic cardiac changes in daunorubicin-induced cardiomyopathy in rabbits. Sb Ved Pr Lek Fak Karlovy Univerzity Hradci Kralove 37: 49-55, 1994.

GRAHAM HK, HORN M, TRAFFORD AW: Extracellular matrix profiles in the progression to heart failure. Acta Physiol 194: 3-21, 2008.

HERMAN EH, ZHANG J, FERRANS VJ: Comparison of the protective effects of desferrioxamine and ICRF-187 against doxorubicin-induced toxicity in spontaneously hypertensive rats. Cancer Chemother Pharmacol 35: 93-100, 1994.

LALU MM, PASINI E, SCHULZE JC, FERRARI-VIVALDI M, FERRARI-VIVALDI G, BACHETTI T, SCHULZ R: Ischemia-reperfusion activates matrix metalloproteinases in the human heart. Eur Heart $J$ 26: 27-35, 2005.

MARIAN AJ: On mice, rabbits, and human heart failure. Circulation 111: 2276-2279, 2005.

MARTY M, ESPIE M, LLOMBART A, MONNIER A, RAPOPORT BL, STAHALOVA V: Dexrazoxane Study Group. Multicenter randomized phase III study of the cardioprotective effect of dexrazoxane (Cardioxane) in advanced/metastatic breast cancer patients treated with anthracycline-based chemotherapy. Ann Oncol 17: 614622, 2006.

PACHER P, LIAUDET L, BAI P, VIRAG L, MABLEY JG, HASKO G, SZABO C: Activation of poly(ADP-ribose) polymerase contributes to development of doxorubicin-induced heart failure. $J$ Pharmacol Exp Ther 300: 862$867,2002$.

PELOUCH V, MILEROVÁ M, OŠŤÁDAL B, HUČÍN B, ŠAMÁNEK M: Differences between atrial and ventricular protein profiling in children with congenital heart disease. Mol Cell Biochem 147: 43-49, 1995.

SCHULZ R: Intracellular targets of matrix metalloproteinase-2 in cardiac disease: rationale and therapeutic approaches. Annu Rev Pharmacol Toxicol 47: 211-242, 2007.

SPINALE FG: Myocardial matrix remodeling and the matrix metalloproteinases: influences on cardiac form and function. Physiol Rev 87: 1285-1342, 2007.

ŠIMU゚NEK T, KLIMTOVÁ I, KAPLANOVÁ J, MAZUROVÁ Y, ADAMCOVÁ M, ŠTĚRBA M, HRDINA R, GERŠL V: Rabbit model for in vivo study of anthracycline-induced heart failure and for the evaluation of protective agents. Eur J Heart Failure 6: 377-387, 2004.

TRUTER SL, CATANZARO DF, SUPINO G, GUPTA A, CARTER J, HERROLD EM, DUMLAO TF, BORER JS: Differential expression of matrix metalloproteinases and tissue inhibitors and extracellular matrix remodelling in aortic regurgitant heart. Cardiology 113: 161-168, 2009. 